\title{
Pescadores y pescadoras, una forma de vida alrededor de los humedales
}

\author{
Sandy Astrid Medina Valdivia
}

\section{The RISC Consortium}

A World Family Portrait is a joint project of the Consortium for Comparative Research on Regional Integration and Social Cohesion (RISC) and Regions $\mathcal{E}$ Cohesion. It aims to promote interdisciplinary and cross-cultural communication through images and essays on the different faces of humanity, including, but not limited to, our similarities and our differences, our strengths and our weaknesses, our hopes and our concerns, our legacies and our aspirations, as well as our interactions with each other and our world. This project seeks to establish a dialogue between human experiences, academic reflections and shared ethics, such as mutual respect, the protection of human dignity and solidarity.

Regions \& Cohesion welcomes submissions of photos and short essays for publication in the World Family Portrait section of the journal. The journal has already published photos and texts on themes such as water, poverty, women, borders, political ideology, democratization, development, religious practices, violence, the environment, and more. Interested contributors should go to the World Family Portrait link at www.risc.lu where texts and photographs can be uploaded along with basic information such as contributor name(s), correspondence address, institutional affiliation and current position. Essays should be approximately 500 words in length. Images should be submitted in a JPEG format at a resolution of approximately $1900 \times 1600$ pixels. All materials must be original. All inquiries and submissions related to the project should be sent via the forms on the RISC Consortium web portal. 


\section{In this issue/En este número}

Regions $\mathcal{E}$ Cohesion is proud to present three photographs by Sandy Astrid Medina Valdivia that describe her research visit to a fishing community in the State of Guerrero in Southern Mexico. "El Veinte" is self-named "a community of fishermen and fisherwomen," which is well recognized in the region. For more than 50 years they have forged a close relationship with the Laguna de Nuxco coastal wetland, as this has provided them with various survival benefits. These images reflect the livelihood of the community: their fishing activity, which in fact represents their major economic income immersed in a sustainable relationship with the environment that shapes their way of life.

\section{Pescadores y pescadoras, una forma de vida alrededor de los humedales}

"El Veinte" es una comunidad de pescadores y pescadoras, así se autonombran y así son reconocidos en la región, ubicada al sur del estado de Guerrero en México. Por más de 50 años estas personas han forjado una estrecha relación con el humedal costero Laguna de Nuxco por los beneficios que les proporciona para sobrevivir. El medio de vida de la comunidad es la actividad pesquera y su economía depende casi al cien por ciento de la venta de productos pesqueros que extraen del humedal. Los primeros pobladores no contaban con energía eléctrica, entonces la pesca se limitaba a lo que podían vender en un solo día. Pescar era un arte ejecutado con cuerdas de nailon tejidas a mano, y era difícil conseguir material flotante para sostenerlos; por otra parte, las redes eran elaboradas de hilo y muy pesadas al mojarse. Esto cambió al inicio de los años setenta con la llegada de la electricidad. La pesca aumentó en cierta medida porque los pescadores podían almacenar el producto en congeladores, además de utilizar redes de nailon que les permitieron capturar un mayor volumen de peces. El reemplazo generacional de pescadores perfeccionó las técnicas de pesca al mismo tiempo que construyó una estructura social con respecto a los papeles que desempeña cada miembro de la familia. En ese sentido, los hombres que son jefes de familia se dedican principalmente a la extracción de productos pesqueros, sin embargo, hay períodos en los que deben buscar una fuente alternativa de ingresos económicos ya que la pesca disminuye. Algunas mujeres también se dedican a pescar, pero la mayoría de ellas son las responsables de transportar y vender los productos pesqueros en diferentes localidades de la región. Las mujeres que 
venden casa por casa son conocidas localmente como "las pescadoras". En ocasiones, camiones de puertos pesqueros vecinos llegan a la localidad para abastecer a las pescadoras ya que los productos del humedal no son siempre suficientes. El deterioro ecológico del humedal ha ocasionado la disminución de los beneficios que los pobladores locales obtienen de este ecosistema. La relación de la comunidad pesquera El Veinte con la Laguna de Nuxco muestra como una comunidad local coexiste y moldea su modo de vida a lo largo de los años en torno a los sistemas naturales que le rodean y de los cuales depende su economía y bienestar. Solo una perspectiva integral de análisis nos permitiría comprenderlos, pero este será el tema de un próximo artículo.

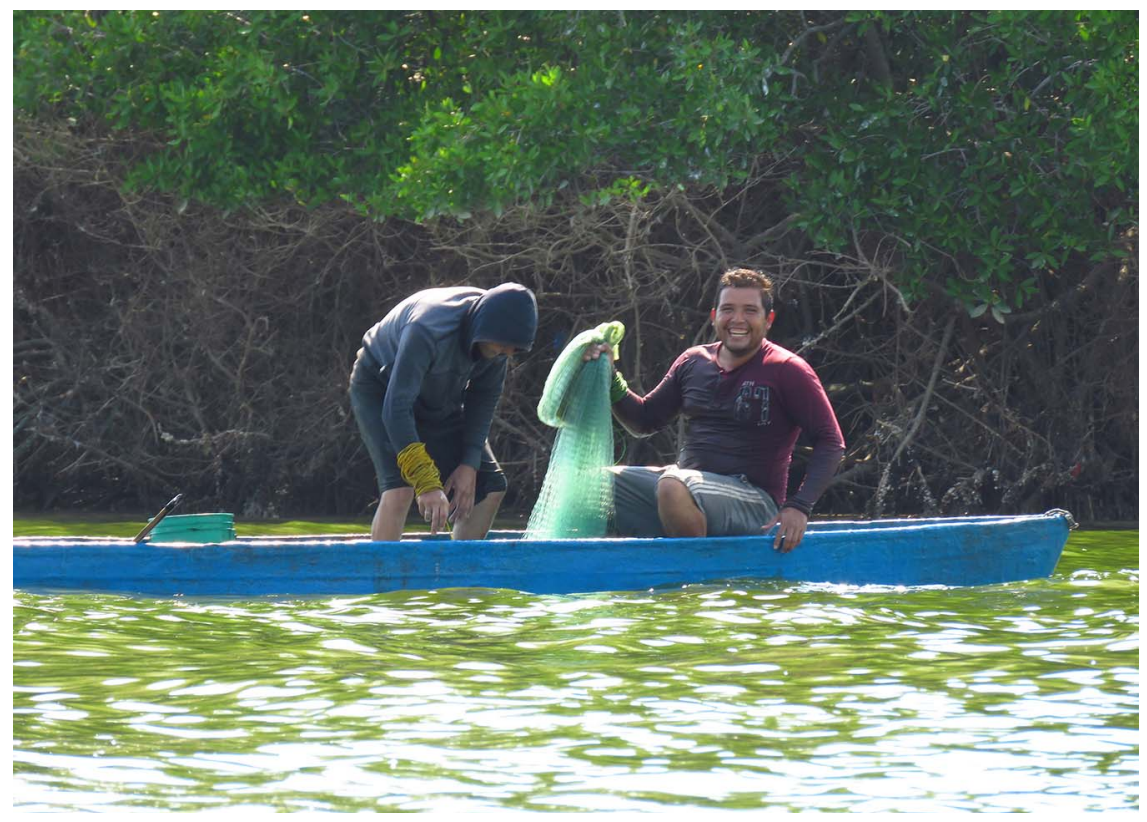

Photo 1 - Jóvenes pescando en el humedal Laguna de Nuxco. El oficio es heredado generación tras generación para quiénes deciden dedicarse a la pesca y permanecer en la comunidad. 


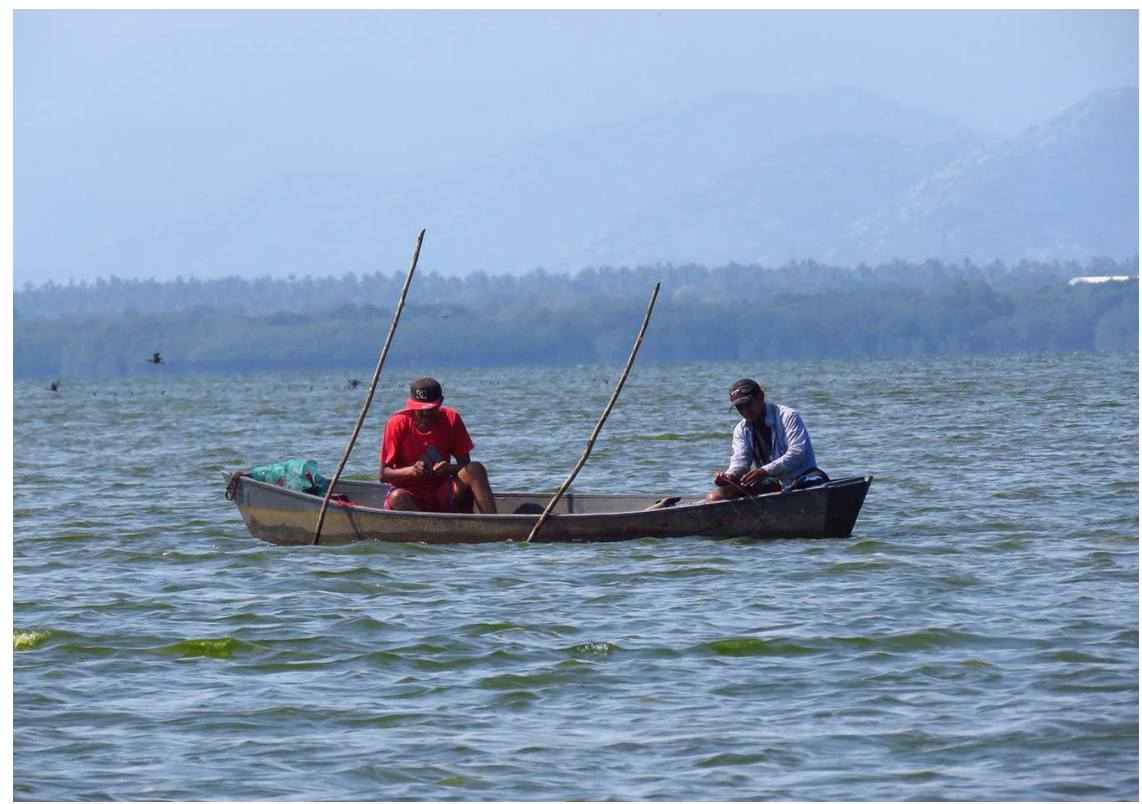

Photo 2 - Las embarcaciones que utilizan los pescadores son de madera y están recubiertas con fibra de vidrio, estas pueden trasladar de dos a tres pescadores.

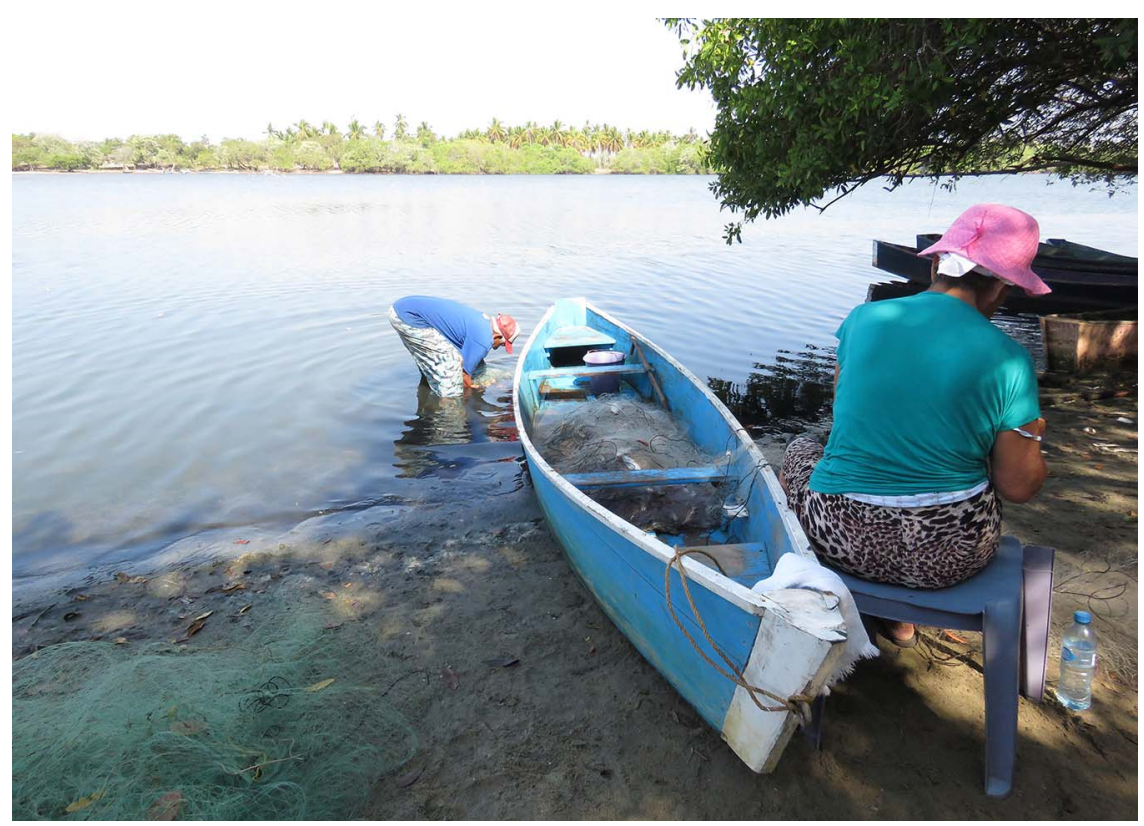

Photo 3 - Una pareja de pescadores revisando la pesca del día, viven junto a la laguna y su bienestar depende de este ecosistema. 
SANDY ASTRID MEDINA VALDIVIA es Bióloga y Maestra en Ciencias por la Universidad Autónoma de Guerrero (UAGRO), con especialidad en Desarrollo Sustentable del Turismo. Actualmente cursa el Doctorado en Ciencias Ambientales de la UAGRO. Colabora con equipos interdisciplinarios y cuenta con amplia experiencia en la consultoría y desarrollo de proyectos ambientales tales como estudios de impacto ambiental, estudios de biodiversidad, conservación de la naturaleza y monitoreo comunitario. Sandy tiene un especial interés por comprender las relaciones de los seres humanos con los ecosistemas y su investigación doctoral en curso aborda los servicios ecosistémicos en humedales costeros de Guerrero, México. 